\title{
Review of: "Therapeutic effects and safety of oral Chinese patent medicine for COVID-19: A rapid systematic review and meta-analysis of randomized controlled trials"
}

Lin Lin

Potential competing interests: The author(s) declared that no potential competing interests exist.

Thank you very much for letting me to review this paper. The paper aimed to review and evaluate the therapeutic effects and safety of oral Chinese patent medicine (CPM) for COVID-19. And the authors conclude that add-on oral CPM can shorten the duration of fever, cough and fatigue, improve the recovery rate of cough and fatigue, and increase the improvement and recovery rate of chest CT manifestations. There are some differences in therapeutic effects among various CPMs for the same COVID-19 outcome. The use of TJQW and LHQG appear not to increase the risk of adverse events, but JHQG may cause mild diarrhea. It is an excellent one. For my opinions, I think the clinic trials relevant to CPM for COVID-19 should include different races in different countries throughout the world. And the mechanisms of CPM treatments for COVID-19 should also be explored. However, I have no other comments. Once again, thanks for inviting me to review this paper. 